\title{
Multisystem inflammatory syndrome in children in South Africa
}

Multisystem inflammatory syndrome in children (MIS-C) is a relatively new clinical entity that has been described in children during the SARS-CoV-2 pandemic. Initial reports emerged around mid-April 2020 and described children presenting with this hyperinflammatory syndrome 4 - 6 weeks after the peak of COVID19 infections. ${ }^{[1-4]}$ These studies were supported by later reports that showed that as the number of COVID-19 infections declined, so did the number of MIS-C cases. ${ }^{[5]}$ The Western Cape, being the initial epicentre of COVID-19 infections within South Africa, had their first MIS-C cases at the beginning of June 2020. ${ }^{[6]}$

Paediatric inflammatory multisystem syndrome temporally associated with SARS-CoV-2 (PIMS-TS) and MIS-C are synonymous names given to this syndrome. ${ }^{[7-9]}$ Despite the differing names and minor differences in case definitions, they include children who present with fever, evidence of inflammation and multi-organ involvement after SARS-CoV-2 exposure. The high incidence of SARS-CoV-2-positive serology as well as the increased markers of hyperinflammation suggest that the syndrome is a post-infectious inflammatory process. ${ }^{[10,11]}$

MIS-C mimics a variety of previously described conditions such as Kawasaki disease, toxic shock and macrophage activation syndromes ${ }^{[4]}$ It may also present with a variety of nonspecific signs requiring medical practitioners and primary healthcare staff to have a high index of suspicion for the condition. Most (70\%) children with MIS-C do not have any co-existing medical conditions and the affected children seem to come disproportionately from black, Afro-Caribbean and Asian backgrounds. ${ }^{[2,3,11-13]}$ Boys seem to be affected slightly more than girls. ${ }^{[10]}$

Children may present with a constellation of signs involving different organ systems. The symptoms can range from mild fever and rash, to shock requiring multi-organ support. ${ }^{[2-4,11,12]}$ The most common presenting feature is fever, and all children had fever at some time in their clinical course..$^{[2,3,11-13]}$

Organ systems commonly involved are gastrointestinal (87\%), mucocutaneous $(73 \%)$, cardiac $(71 \%)$ and neurological (22\%). ${ }^{[10]}$ Respiratory involvement is rare. ${ }^{[4]}$

Gastrointestinal manifestations range from nausea, vomiting and nonspecific abdominal pain to an acute abdomen mimicking acute appendicitis. ${ }^{[14]} \mathrm{A}$ few children have had negative laparotomies for appendicitis, and therefore the diagnosis of MIS-C must be considered in children presenting with an acute appendicitis during these pandemic times. Mucocutaneous manifestations include conjunctivitis, stomatitis, skin peeling and a variety of nonspecific rashes. Though neurological signs are less frequently described, children can present with headache, meningism, seizures and encephalopathy. ${ }^{[2,3,11,12,14]}$ Despite children not presenting with any signs of cardiac involvement, abnormalities of the coronary arteries have been found on echocardiography. Troponin $T$ and B-natriuretic peptide levels are also raised in a significant proportion of children. ${ }^{[2,3,10,12]}$

Laboratory testing in patients with MIS-C reveals significant elevation of inflammatory markers such as C-reactive protein, erythrocyte sedimentation rate, procalcitonin and ferritin. Children with MIS-C may also have a neutrophilia, lymphopenia and low to normal platelet counts. Many children also have elevated $D$-dimer and fibrinogen levels. ${ }^{[4,10,14]}$
The majority $(80 \%)$ of children with MIS-C in North America were cared for in a paediatric ICU, while only $25 \%$ of children in a Pakistani cohort received ICU care. ${ }^{[2,3,13]}$ The reported mortality in children with MIS-C is 2 - 3\% for high-income countries, while the mortality rate in the Pakistani cohort was $13 \% \cdot{ }^{[2,3,11-13]}$ Even though paediatric critical care resources in South Africa (SA) are limited, ${ }^{[15]}$ it is recommended that children with suspected MIS-C be referred to tertiary centres that have paediatric cardiology and paediatric critical care services. It is mandatory for these patients to have an echocardiographical assessment.

Most children with MIS-C have been managed supportively with intravenous immunoglobulin (IVIG) and glucocorticoids. Very few children have required anti-tumour necrosis factor, and interleukin-1 (IL-1) or IL-6. ${ }^{[2,3,11-13]}$ There is substantial variability and a lack of consensus on the use of anticoagulants. Low-dose aspirin has also been used until such time as the follow-up echocardiogram excludes any persisting coronary artery abnormality. ${ }^{[4]}$

\section{MIS-C SA Working Group}

A countrywide working group of paediatric specialists has been formed with the aim of supporting clinicians managing children with MIS-C. As MIS-C is a new condition, we aim to pool expertise from different centres across the country. Participation in the group is open to all practitioners across the country with an interest in MIS-C.

Emails should be sent to southafricamisc@gmail.com for any queries or questions about MIS-C as well as the notification process. Support has also been provided to our adult colleagues, as many paediatric wards in SA only admit children up to 13 years of age and a number of older adolescents have presented to adult physicians with MIS-C. ${ }^{[16]}$

From September 2020, MIS-C is a notifiable medical condition (NMC) in SA and utilises the WHO case definition ${ }^{[17]}$ which can be found at https://www.nicd.ac.za/wp-content/uploads/2020/09/ MIS_C-Case-Def_09-Sept-2020.pdf. Importantly, even though MIS-C is a COVID-19-related condition, for the notification process it is not listed under the COVID-19 section but instead appears towards the end of the list of general notifiable conditions. Together with the notification process, a case report form (CRF) needs to be completed for all probable and confirmed MIS-C cases. All of this information can be found on the NICD website at https://www.nicd. ac.za/nmc-overview/nmc-covid-19-documents/. ${ }^{[18]}$ Notification can be done online or via a mobile app.

In a report from the MIS-C working group, dated 5 October 2020, there were $\sim 126$ anecdotal cases of MIS-C diagnosed and managed in SA, whereas until that date only six cases had been captured by the NMC notification process. This is probably an under-representation of the true number of cases, as most clinicians are still unfamiliar with the process. There is an urgent need to gather prospective reports of probable and confirmed cases and support the collection of retrospective data to inform the true burden of MIS-C in SA.

If the number of COVID-19 infections is kept at bay, MIS-C is likely to become a rare disease in SA; but, if there is a large uncontrolled rise in the number of infections, MIS-C is perhaps going to be a disease that paediatricians will become well accustomed to. ${ }^{[5]}$ All child and adolescent health practitioners are therefore encouraged to 
familiarise themselves with the case definitions of MIS-C, refer and discuss cases with specialist teams, and notify all suspected cases.

\section{A Salloo \\ Department of Critical Care, Chris Hani Baragwanath Academic Hospital and Division of Critical Care; and Department of Paediatrics and Child Health, University of the Witwatersrand, Johannesburg, South Africa \\ asma.salloo@wits.ac.za}

1. Riphagen S, Gomez X, Gonzalez-Martinez C, Wilkinson N, Theocharis P. Hyperinflammatory shock in children during COVID-19 pandemic. Lancet 2020;395(10237):1607-1608. https://doi.org/10.1016/s01406736(20)31094-1

2. Dufort EM, Koumans EH, Chow EJ, et al. Multisystem inflammatory syndrome in children in New York State. N Eng J Med 2020;18;383(4):347358. https://doi.org/10.1056/NEJMoa2021756

3. Feldstein LR, Rose EB, Horwitz SM, et al. Multisystem inflammatory syndrome in U.S. children and adolescents. N Eng J Med 2020;383(4):334346. https://doi.org/10.1056/NEJMoa2021680

4. Jiang L, Tang $\mathrm{K}$, Levin $\mathrm{M}$, et al. COVID-19 and multisystem inflammatory syndrome in children and adolescents. Lancet Infect Dis 2020;20(11):e276-e288. https://doi.org/10.1016/S1473-3099(20)30651-4

5. Multisystem inflammatory syndrome in children in the United States. N Eng J Med 2020;383(18):1793-1796. https://doi.org/10.1056/NEJMc2026136

6. Webb K, Abraham DR, Faleye A, McCulloch M, Rabie H, Scott C. Multisystem inflammatory syndrome in children in South Africa. Lancet Child Adolesc Health 2020;4(10):e38. https://doi.org/10.1016/S23524642(20)30272-8

7. COVID-19-Paediatric-multisystem-inflammatory syndrome-20200501. pdf. https://www.rcpch.ac.uk/sites/default/files/2020-05/COVID-19Paediatric-multisystem-\%20inflammatory\%20syndrome-20200501.pdf (accessed 18 September 2020).

8. HAN Archive - 00432 | Health Alert Network (HAN) 2020. https:// emergency.cdc.gov/han/2020/han00432.asp (accessed 18 September 2020).
9. World Health Organization. Multisystem inflammatory syndrome in children and adolescents with COVID-19. https://www.who.int/ publications-detail-redirect/multisystem-inflammatory-syndrome-inchildren-and-adolescents-with-covid-19 (accessed 18 September 2020).

10. Abrams JY, Godfred-Cato SE, Oster ME, et al. Multisystem inflammatory syndrome in children associated with severe acute respiratory syndrome coronavirus 2: A systematic review. J Pediatr 2020;226:45-54.e1. https://doi. org/10.1016\%2Fj.jpeds.2020.08.003

11. Whittaker E, Bamford A, Kenny J, et al. Clinical characteristics of 58 children with a pediatric inflammatory multisystem syndrome temporally associated with SARS-CoV-2. JAMA 2020;324(3):259-269. https://doi.org/10.1001/ jama.2020.10369

12. Davies P, Evans C, Kanthimathinathan HK, et al. Intensive care admissions of children with paediatric inflammatory multisystem syndrome temporally associated with SARS-CoV-2 (PIMS-TS) in the UK: A multicentre observational study. Lancet Child Adolesc Health 2020;4(9):669-677. https:// doi.org/10.1016/S2352-4642(20)30215-7

13. Sadiq M, Aziz OA, Kazmi U, et al. Multisystem inflammatory syndrome associated with COVID-19 in children in Pakistan. Lancet Child Adolesc Health 2020;4(10):e36-e37. https://doi.org/10.1016/s2352-4642(20)30256-x

14. Nakra NA, Blumberg DA, Herrera-Guerra A, Lakshminrusimha S. Multisystem inflammatory syndrome in children (MIS-C) following SARS-CoV-2 infection: Review of clinical presentation, hypothetical pathogenesis, and proposed management. Children 2020;7(7):69. https://doi.org/10.3390/ children7070069

15. Coovadia H, Jewkes R, Barron P, Sanders D, McIntyre D. The health and health system of South Africa: Historical roots of current public health challenges. Lancet 2009;374(9692):817-834. https://doi.org/10.1016/s01406736(09)60951-x

16. Parker A, Louw EH, Lalla U, et al. Multisystem inflammatory syndrome in adult COVID-19 patients. S Afr Med J 2020;110(10):957-958. https://doi. org/10.7196\%2FSAMJ.2020.v110i10.15244

17. MIS_C-Case-Def_09-Sept-2020.pdf. https://www.nicd.ac.za/wp-content/uploads/2020/09/MIS_C-Case-Def_09-Sept-2020.pdf (accessed 23 September 2020).

18. MIS-C-SOP_09-September-2020.pdf. https://www.nicd.ac.za/wp-content/uploads/2020/09/MIS-C-SOP_09-September-2020.pdf (accessed 12 November 2020).

S Afr J Child Health 2020;14(4):169-170. https://doi.org/10.7196.SAJCH.2020.v14i4.1850 